\title{
Two isoforms of human SPRR3 are highly represented in human pre-term newborn saliva
}

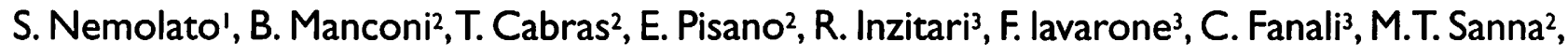 \\ C. Romagnoli4, G. Faa', M. Castagnola ${ }^{3}$, I. Messana ${ }^{2}$ \\ ' Dipartimento di Citomorfologia, Università di Cagliari, Italy \\ 2 Dipartimento di Scienze Applicate ai Biosistemi, Università di Cagliari, Italy \\ ${ }^{3}$ Istituto di Biochimica e Biochimica Clinica, Università Cattolica, Rome, Italy \\ ${ }^{4}$ Dipartimento di Pediatria, Divisione di Neonatologia, Università Cattolica, Rome, Italy
}

During a proteomic study of human pre-term newborn saliva we have observed great amounts of two proteins that have never been detected before in adult saliva [I]. In the present study, by using different ESI-MS approaches and cDNA sequencing, we demonstrated that they correspond to two isoforms of the small proline-rich protein 3 (SPRR3).

Deconvolution of the ESI-MS spectra of the peak eluting between 26.6 and $27.6 \mathrm{~min}$ in the RP-HPLC-ESI-MS profile of human pre-term newborn saliva revealed the average molecular mass (Mav) values $17239 \pm 3 \mathrm{Da}$ and $18065 \pm 3 \mathrm{Da}$ in 9 samples of human pre-term newborn saliva, a Mav value $17239 \pm 3 \mathrm{Da}$ in 4 samples and a Mav value $18065 \pm 3$ $\mathrm{Da}$ in 2 samples. MALDI-TOF and MS/MS analysis of tryptic digests allowed identifying the proteins as SPRR3, previously described in two isoforms [2, 3, Swiss-Prot code Q9UBC9,A5YKK8 respectively], differing for an octapeptide repeat and for the substitution $\mathrm{Leu}_{149} \rightarrow \mathrm{Val}$. cDNA amplification of RNA extracts from oral mucosa, parotid and submandibular gland samples obtained at fetal autopsy provided two nucleotide sequences in agreement with those reported in the literature. CDNA sequencing also showed that the two proteins differ for one of the 14 approximate octapeptide tandem repeats present in the isoform with higher Mav value, with sequence GCTKVPEP, and for the substitution Leu $\rightarrow$ Val at position 149 and I4I in the higher and lower Mav value isoforms, respectively. However, cDNA amplification did not allow to clarify if the protein found in saliva originated from cellular shedding of the epithelium and/or secretion. Experimental Mav values of the intact proteins did not correspond to those available in databank for human SPRR3. The difference is due to the removal of initiator methionine and the presence of $\mathrm{N}$ terminal acetyl group, the latter also confirmed by incubation with $25 \%$ TFA at $55^{\circ} \mathrm{C}$ for $60 \mathrm{~min}$. The two post translational modifications have not been previously described for these proteins.

\section{References}

[1] Messana I., Cabras T., Pisano E., Sanna M.T., Olianas A., Manconi B., Pellegrini M., Paludetti G., Scarano E., Fiorita A., Agostino S., Contucci A.M., Calò L., Picciotti P.M., Manni A., Bennick A.,Vitali A., Fanali C., Inzitari R., Castagnola M. 2008. Trafficking and post-secretory events responsible for the formation of secreted human salivary peptides.A proteomic approach. Molecular \& Cellular Proteomics 7(5): $911-926$.

[2] Gibbs S., Fijneman R.,Wiegant J., van Kessel A.G., van De Putte P., Backendorf C. 1993. Molecular characterization and evolution of the SPRR family of keratinocyte differentiation markers encoding small proline-rich proteins. Genomics 16(3): 630-637.

[3] Marenholz I., Zirra M., Fischer D.F., Backendorf C., Ziegler A., Mischke D. 200I. Identification of Human Epidermal Differentiation Complex (EDC)-Encoded Genes by Subtractive Hybridization of Entire YACs to a Gridded Keratinocyte cDNA Library. Genome Res. I I(3): 341-355.

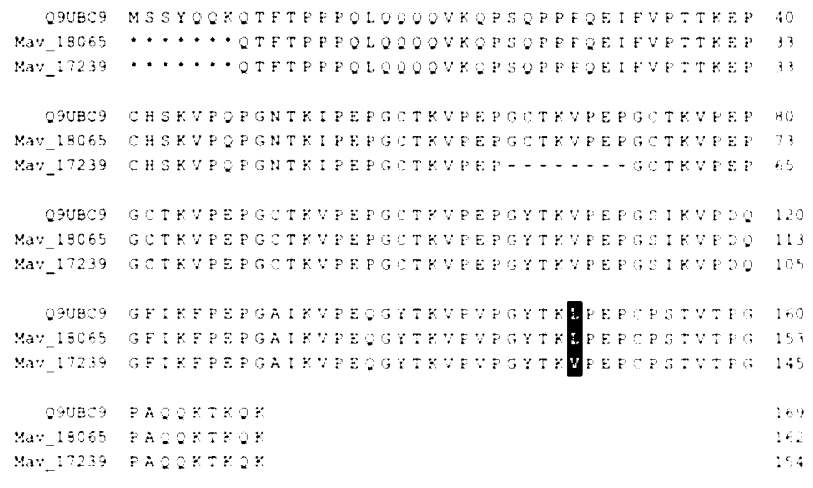

Figure - Alignment of SPRR3 sequence (Q9UBC9) with the CDNA derived amino-acid sequences of the two isoforms of human SPRR3 from fetal autoptic parotid gland.Asteriscs indicate the first 7 residues not determined in our sequences. 